\title{
THE HARD FACTS
}

For women worldwide, breast cancer is the most common cancer diagnosed and has the highest death toll. With improvements in screening and treatments over the past 50 years, more women are living longer, but the numbers reveal some tough challenges. By Amy Maxmen.

Age-standardized breast cancer incidence rates vary internationally by more than 13 -fold. Disease diagnoses are more common in industrialized, Western countries that have high rates of screening; extremely low incidence rates in many developing countries in part reflect low screening rates and incomplete reporting.

\begin{tabular}{|l|l|l}
\hline$>72.3$ & $25.9-36.2$ & Cases \\
\hline $50.3-72.2$ & $17.2-25.8$ & per \\
\hline $36.3-50.2$ & $\leq 17.1$ & 100,000 \\
\hline
\end{tabular}
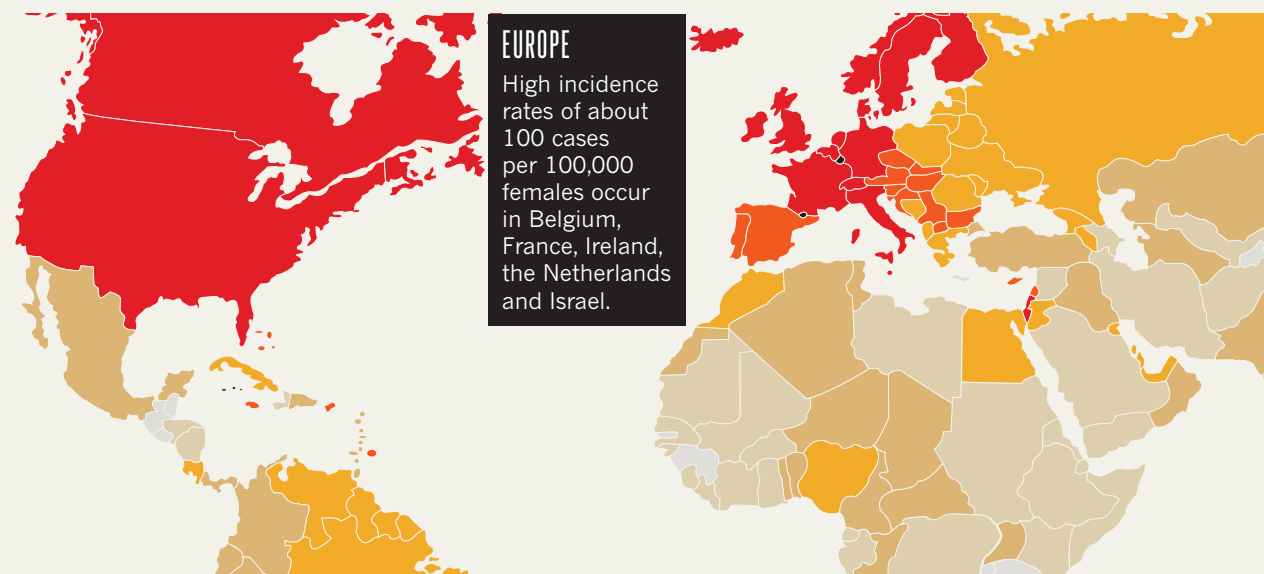

SCREENING

Mammography

screening rates vary

widely, ranging from

$12 \%$ in Turkey and

$17 \%$ in Mexico, to

more than $80 \%$

in Finland, the

Netherlands and

the United States.

\section{DEVELOPING COUNTRIES \\ Rising rates of \\ breast cancer \\ diagnoses in many \\ African and Asian \\ countries might \\ reflect increases in \\ screening, and \\ possibly rising rates \\ of obesity, dietary \\ changes and \\ delayed childbirth.}

\section{EAST ASIA \\ South Korea and Japan have low rates of breast cancer mortality (6.1 and 10.8 per 100,000) relative to other industrialized countries, but unlike elsewhere, those rates are rising.}

\section{GROWING PAINS}

Women in the United States have a roughly $12 \%$ chance of developing breast cancer at some point in their life. But that risk increases when breast cancer runs in the family. Hereditary mutations in the tumour-suppressor genes $B R C A 1$ and $B R C A 2$ raise an individual's risks of developing breast cancer to $60 \%$ and $85 \%$, respectively. Disregarding heritable factors, the likelihood of breast cancer through the decades is as follows:

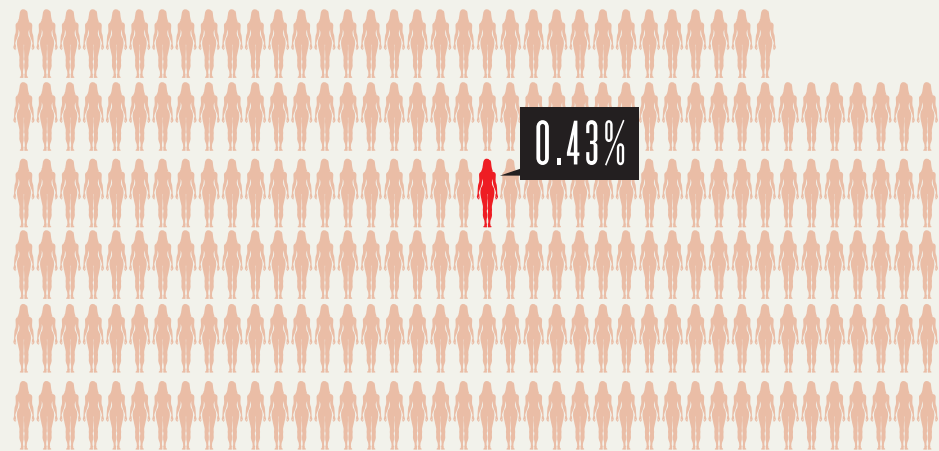

Women age 30-39: 1 in 233 people

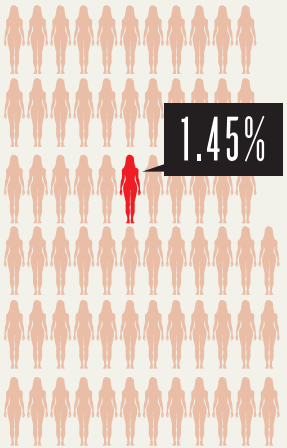

Age 40-49

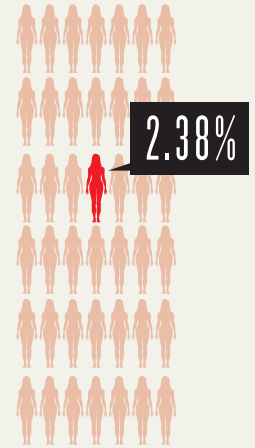

Age 50-59
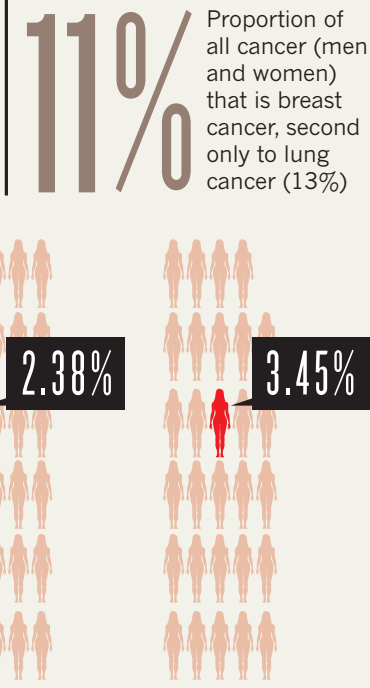

Age 60-69 


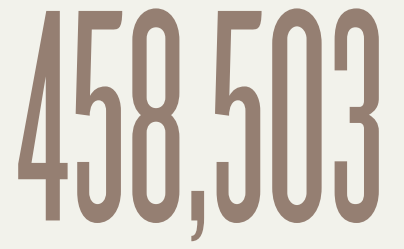

The number of women who died from breast cancer worldwide in 2008

\section{ADVANCES FOR SOME}

Increased screening and improved therapies have resulted in lower mortality rates in some population segments. But, although the decline is steep for white women, it is more gradual for African-Americans. The reasons range from inadequate access to healthcare services to biological differences that might reflect genes or environmental exposures.

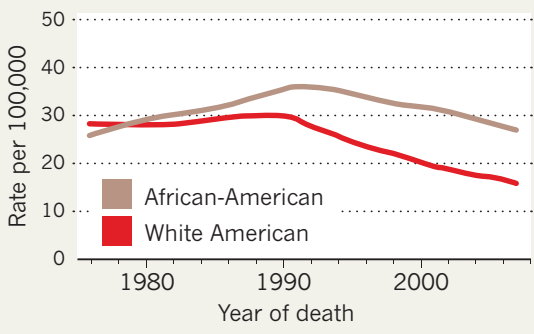

\section{SURVIVAL FACTORS}

Many factors influence a woman's chance of survival, including how early the tumour is detected and the molecular profile of the tumour. Even so, women diagnosed now are much more likely to survive than women in decades past.

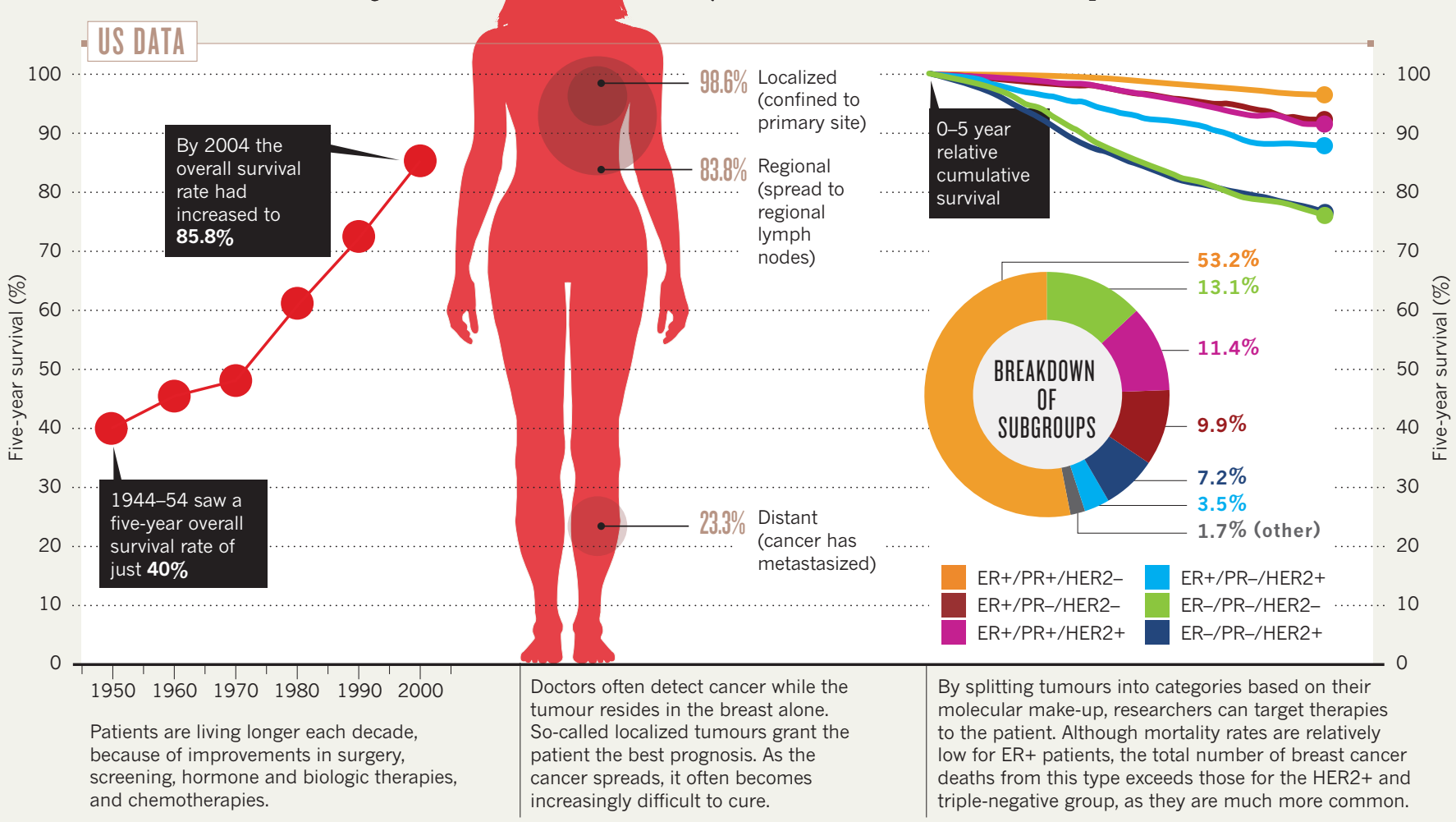

\section{THE MALE FACTOR}

Male breast cancer accounts for about $1 \%$ of all breast cancers. Although the incidence appears to be increasing, death rates have decreased by $3.3 \%$ a year since 2000 .

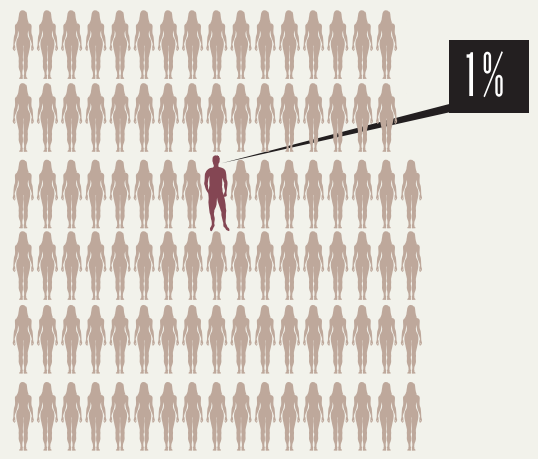

All breast cancer cases

\section{BITTERSWEET SUCCESS}

The cost of breast cancer is expected to rise as the population ages and patients live longer because of better - and more expensive - drugs. Because breast cancer is highly prevalent, it might have the highest price tag of any cancer by 2020. The chart projects the costs assuming a $2 \%$ increase in the initial treatment phase and a $2 \%$ increase in the final year of life.

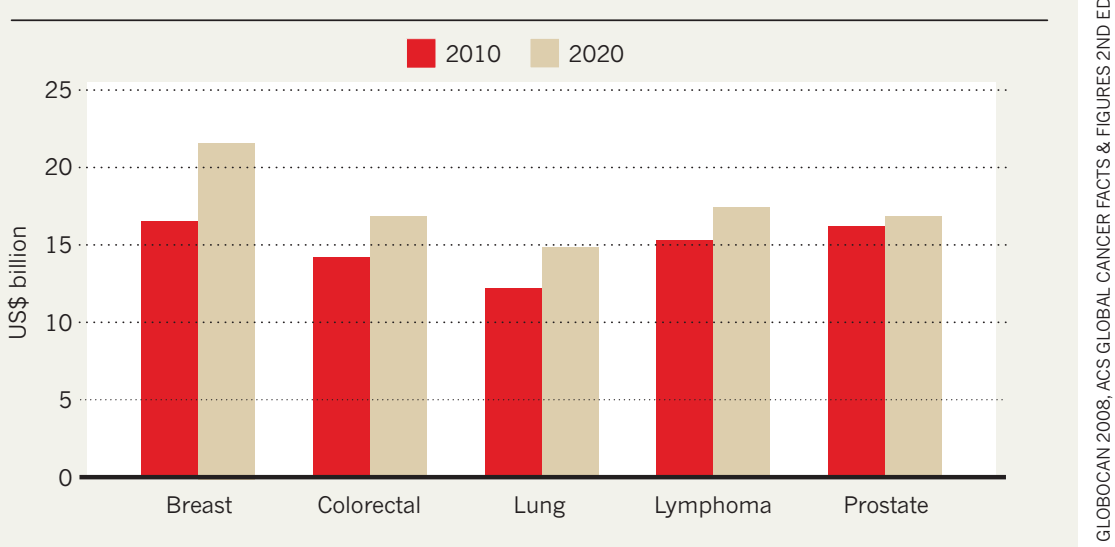

